\title{
A PERTURBATION THEOREM FOR COMPLETE SETS OF COMPLEX EXPONENTIALS
}

\author{
ROBERT M. YOUNG
}

\begin{abstract}
The purpose of this note is to show that the completeness of a set of complex exponentials $\left\{e^{i \lambda_{n} t}\right\}$ in $L^{2}(-\pi, \pi)$ is preserved whenever the $\lambda_{n}$ are subjected to a suitable "lifting".
\end{abstract}

There is an extensive literature on the completeness of sets of complex exponentials $\left\{e^{i \lambda_{n} t}\right\}$ (see, for example, [1]-[8], and the references therein). In this note, we show that completeness is preserved in $L^{2}(-\pi, \pi)$ whenever the $\lambda_{n}$ are subjected to a suitable "lifting".

THEOREM. Let $\left\{\lambda_{n}\right\}$ and $\left\{\mu_{n}\right\}$ be two sequences of points lying in a fixed horizontal strip and suppose that $\operatorname{Re} \lambda_{n}=\operatorname{Re} \mu_{n}$. If $\left\{e^{i \lambda_{n} t}\right\}$ is complete in $L^{2}(-\pi, \pi)$, then so too is $\left\{e^{i \mu_{n} t}\right\}$.

Proof. By making a suitable translation, we may assume that $\lambda_{n} \mu_{n} \neq 0$. Suppose that the set $\left\{e^{i \mu_{n} t}\right\}$ is not complete in $L^{2}(-\pi, \pi)$. Then there exists a function $f_{0}$ in $L^{2}(-\pi, \pi)$ not equivalent to zero such that

$$
\int_{-\pi}^{\pi} f_{0}(t) e^{i \mu_{n} t} d t=0 \quad(n=1,2, \ldots) .
$$

Let us denote by $H$ the Paley-Wiener space of entire functions $F$ of exponential type $\pi$ for which

$$
\|F\|=\left\{\int_{-\infty}^{\infty}|F(x)|^{2} d x\right\}^{1 / 2}<\infty .
$$

If we set

$$
F_{0}(z)=\int_{-\pi}^{\pi} f_{0}(t) e^{i z t} d t
$$

then $F_{0}$ belongs to $H$, is not identically zero, and $F_{0}\left(\mu_{n}\right)=0$ for each $\mu_{n}$. We may suppose in addition that $F_{0}(0)=1$. This is clear if $F_{0}(0) \neq 0$, while if $F_{0}$ has a zero of order $m$ at the origin, then dividing $F_{0}$ by a suitable multiple of $z^{m}$ produces the desired function.

Let

$$
F_{n}(z)=F_{0}(z) \prod_{k=1}^{n} \frac{z-\lambda_{k}}{z-\mu_{k}} \frac{\mu_{k}}{\lambda_{k}} \quad(n=1,2, \ldots) .
$$

Received by the editors August 6, 1975.

AMS (MOS) subject classifications (1970). Primary 42A64; Secondary 46E30.

Key words and phrases. Complex exponentials, Paley-Wiener space.

(c) American Mathematical Society 1976 
Then $F_{n} \in H, F_{n}(0)=1$, and $F_{n}\left(\lambda_{k}\right)=0(k=1,2, \ldots, n)$. We are going to show that the norms $\left\|F_{n}\right\|$ are uniformly bounded in $n$. By the Paley-Wiener representation for functions in $H$, we have

$$
F_{n}(z)=\int_{-\pi}^{\pi} f_{n}(t) e^{i z t} d t \quad \text { with } f_{n} \text { in } L^{2}(-\pi, \pi) .
$$

But then

$$
\int_{-\pi}^{\pi} f_{n}(t) e^{i z t} d t=\frac{z-\lambda_{n}}{z-\mu_{n}} \frac{\mu_{n}}{\lambda_{n}} \int_{-\pi}^{\pi} f_{n-1}(t) e^{i z t} d t,
$$

and it was shown by Levinson $[2$, p. 10] that

$$
f_{n}(t)=\frac{\mu_{n}}{\lambda_{n}}\left[f_{n-1}(t)+i\left(\lambda_{n}-\mu_{n}\right) e^{-i \mu_{n} t} \int_{-\pi}^{\pi} f_{n-1}(x) e^{i \lambda_{n} x} d x\right] .
$$

Since $\sup \left|\lambda_{n}-\mu_{n}\right|<\infty$, obvious estimates yield

$$
\left\|f_{n}\right\| \leqslant A\left|\mu_{n} / \lambda_{n}\right|\left\|f_{n-1}\right\|,
$$

where $A$ is independent of $n$. Therefore,

$$
\left\|f_{n}\right\| \leqslant A\left\|f_{0}\right\| \prod_{k=1}^{n}\left|\frac{\mu_{k}}{\lambda_{k}}\right|
$$

and it remains only to estimate the products $\prod_{k=1}^{n}\left|\mu_{k} / \lambda_{k}\right|$. From the conditions on $\left\{\lambda_{n}\right\}$ and $\left\{\mu_{n}\right\}$ it follows that

$$
\left|\frac{\mu_{k}}{\lambda_{k}}\right|^{2}=1+\frac{\left(\operatorname{Im} \mu_{k}\right)^{2}-\left(\operatorname{Im} \lambda_{k}\right)^{2}}{\left(\operatorname{Re} \lambda_{k}\right)^{2}+\left(\operatorname{Im} \lambda_{k}\right)^{2}} \leqslant 1+B /\left|\lambda_{k}\right|^{2},
$$

where $B$ is independent of $k$. Therefore, for all $n$,

$$
\begin{aligned}
\prod_{k=1}^{n}\left|\frac{\mu_{k}}{\lambda_{k}}\right| & \leqslant \prod_{k=1}^{n}\left(1+\frac{B}{\left|\lambda_{k}\right|^{2}}\right)^{1 / 2} \leqslant \prod_{k=1}^{\infty}\left(1+\frac{B}{\left|\lambda_{k}\right|^{2}}\right)^{1 / 2} \\
& \leqslant \exp \left[\frac{B}{2} \sum_{k=1}^{\infty} \frac{1}{\left|\lambda_{k}\right|^{2}}\right] .
\end{aligned}
$$

Now, $F_{0}$ is entire of exponential type, and hence of order no larger than 1. Therefore, its exponent of convergence is also at most 1 , and in particular, the series $\sum 1 /\left|\mu_{n}\right|^{2}$ is convergent. It follows that the series $\sum 1 /\left|\lambda_{n}\right|^{2}$ is also convergent, and we conclude that sup $\left\|f_{n}\right\|<\infty$. Since the Fourier transform is an isometry, the norms $\left\|F_{n}\right\|$ are uniformly bounded. But $H$ is a functional Hilbert space, and therefore a subsequence of $\left\{F_{n}\right\}$ will converge weakly to a function $G$ in $H$ for which $G\left(\lambda_{n}\right)=0(n=1,2, \ldots)$ and $G(0)=1$. Writing

$$
G(z)=\int_{-\pi}^{\pi} g(t) e^{i z t} d t,
$$

with $g$ in $L^{2}(-\pi, \pi)$, we conclude that the sequence $\left\{e^{i \lambda_{n} t}\right\}$ is not complete in 
$L^{2}(-\pi, \pi)$, contrary to assumption. The contradiction establishes the theorem.

ADDED IN PROOF. Ray Redheffer has informed me that this result, with a different proof, appeared in J. Elsner's doctoral dissertation (Georg-August Univ., Göttingen, 1969).

\section{REFERENCES}

1. A. Beurling and P. Malliavin, On the closure of characters and the zeros of entire functions, Acta Math. 118 (1967), 79-93.

2. N. Levinson, Gap and density theorems, Amer. Math. Soc. Colloq. Publ., vol. 26, Amer. Math. Soc., Providence, R.I., 1940. MR 2, 180.

3. W. Luxemburg, Closure properties of sequences of exponentials, Topics in Analysis, Lecture Notes in Math., vol. 419, Springer-Verlag, New York, 1974, pp. 268-283.

4. R. Paley and N. Wiener, Fourier transforms in the complex domain, Amer. Math. Soc. Colloq. Publ., vol. 19, Amer. Math. Soc., Providence, R.I., 1934.

5. R. M. Redheffer, Elementary remarks on completeness, Duke Math. J. 35 (1968), 103-116. MR 37 \#687.

6. - Two consequences of the Beurling-Malliavin theory, Proc. Amer. Math. Soc. 36 (1972), 116-122. MR 48 \#801.

7. L. Schwartz, Etudes des sommes d'exponentielles, 2ième éd., Publ. Inst. Math. Univ. Strasbourg, V, Actualités Sci. Indust., no. 959, Hermann, Paris, 1959. MR 21 \#5116.

8. R. Young, On perturbing bases of complex exponentials in $L^{2}(-\pi, \pi)$, Proc. Amer. Math. Soc. 53 (1975), 137-140.

Department of Mathematics, Oberlin College, Oberlin, Ohio 44074 